Article

\title{
Dynamics Modelling and Simulation for Deployment Characteristics of Mesh Reflector Antennas
}

\author{
Xin Jiang ${ }^{1}$ and Zhengfeng Bai ${ }^{2, *(B)}$ \\ 1 Department of Aerospace Engineering, Harbin Institute of Technology, No. 92, West Dazhi Street, \\ Harbin 150001, China; 19B318010@hit.stu.edu.cn \\ 2 Department of Mechanical Engineering, Harbin Institute of Technology, Weihai, No. 2, Wenhua West Road, \\ Weihai 264209, China \\ * Correspondence: zfbai@hit.edu.cn
}

Received: 14 October 2020; Accepted: 5 November 2020; Published: 6 November 2020

check for updates

\begin{abstract}
The dynamics of mesh reflectors are characterized by large deformation when antennas undergo a large motion in the deployment process. In this work, absolute node coordinate formulation (ANCF), with the merit of precise description of large deformation, is employed to consider the flexibility of the reflector net. From a practical perspective, the effect of the torsional spring is incorporated into the dynamic governing equations by an equivalent mechanical model. Deployment simulation of the mesh reflector antennas is performed by a numerical procedure. It can be found that with the action of a degenerative driven force caused by the friction effect, the deployed process of antennas exhibits a character of asynchronous deployment. Additionally, a significant increase in tension during the final period of deployment has an intense influence on smooth deployment.
\end{abstract}

Keywords: mesh reflector antenna; large deformation; absolute node coordinate formulation (ANCF); torsional spring; dynamics characteristics

\section{Introduction}

Inherited with merits of large aperture, big shrinkage ratio, and simple topological structure [1,2], the deployable hoop truss antenna is a significant form of satellite antenna [3]. Furthermore, better accuracy and heat stability are presented in the hoop truss antenna compared with other types. As depicted in Figure 1, the deployable hoop truss consists of front and rear nets, a deployable truss, and a vertical cable. The shape configuration and stiffness of the reflector net are constructed with tensile cable. During the initial state of launch of the antenna, the deployable truss is folded in a shrinkage state due to the capacity of the launch vehicle. As the satellite enters orbit, deployment of the truss is initiated under the action of a torsional spring in the synchronous joint to a certain deployed angle. Then, the driving motor drives the cable penetrating through the inclined rod to continue the following expansion of the antenna. A final deployed configuration is locked by the locking mechanism between the thin inclined rod and thick inclined rod [4].

Dynamics simulation of the hoop truss antenna involved complex dynamic behaviors during on-orbit deployment [5], such as large scale numerical computation, coupling characteristics of the rigid-flexible system, and nonlinearity of clearance impact [6,7], which pose a challenge to the structural design and controller development of the antenna. Many research studies have been devoted to the investigation on deployment dynamics of the truss antenna in terms of ground test and numerical simulation. Jin et al. conducted a deployment analysis in terms of numerical computation and experiment verification [8]. Chen et al. studied the dynamics of truss without a cable net based on the experimental setup [9]. A ground experiment performed by Li et al. indicated that the attenuation of driving forces caused by friction effect would induce asynchronous deployment [10]. 


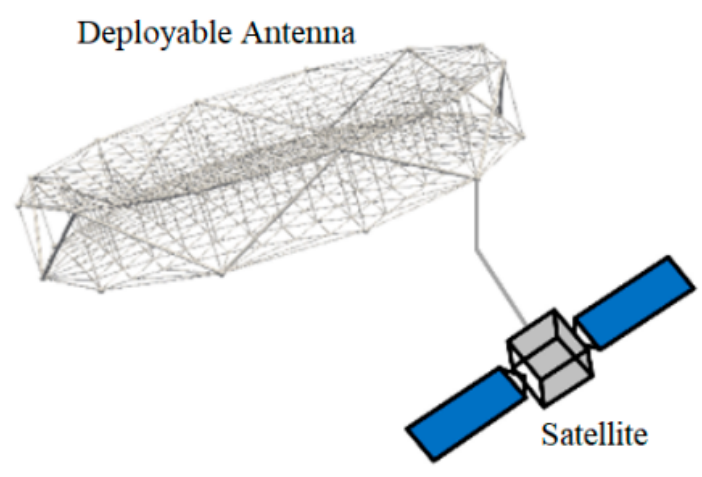

Figure 1. Deployable truss antenna.

By contrast with the high economic cost of the ground test, numerical simulation on deployment dynamics provides access to a reliable and economic design of the deployable truss antenna. Deployment analysis of a ring truss under a pre-designed trajectory was researched [11]. Several mechanical models of deployable antennas have been proposed, such as finite element [12], elastic catenary element [13], and spring-damp element [14,15], to predict the dynamic behaviors of large deformation, contact impact, slack, and wrapping during deployment. Despite the fact that a state of slack or tensile can be captured by the elastic catenary element [13], the condition of the quasi-static process was needed. However, the adopted assumption of finite rotation in conventional finite element could not precisely describe the large deformation [16] of the mesh reflector. Under the assumption of small deformation [17], the influence of truss flexibility was considered in the synchronous deployment of an AstroMesh reflector. Ambrosio et al. analyzed the optimal deployment of a radar antenna using the finite element method under the assumption of infinite small rotations [18], in which the mode component synthesis was adopted to reduce computational cost. A modified deployment planning method was proposed in [17], in which the tension of the cable net is acquired by a simplified model.

To describe the large deformation of the flexible body undergoing large scale motion, two main methodologies were developed: co-rotational coordinate formulation [19] and global coordinate formulation. In detail, the latter can be categorized as theory of geometric accuracy [20], isogeometric analysis [21], and absolute nodal coordinate formulation (ANCF) [22]. Due to the advantage of the gradient vector of a particle being assumed as node coordinates, the dynamic behavior of large deformation can be described precisely by an ANCF element. Additionally, the mass matrix obtained by ANCF possesses the metric of constant and the Coriolis item vanishes. Based on the ANCF beam element, Li investigated the dynamic characteristics of a deployable truss under the action of heat flow [23]. Tao et al. [24] discussed the asynchronous deployment of a hoop truss considering the role of torsional spring. Furthermore, discretizing the mesh reflector with an ANCF element, Liu [25] researched the asynchronous deployment of a hoop truss antenna with a degenerative driving force applied on the inclined rod. An optimal deployment based on fifth-order polynomial programming using ANCF was presented in [26]. In addition, a strategy of parallel computation [27] was introduced to enhance the computation efficiency involving ANCF-based dynamic analysis. A variable-length element is developed by the arbitrary Lagrangian-Euler description to investigate the deployment phenomenon of asynchronism of the hoop truss antenna [28].

From a practical perspective for application in engineering, the deployed angle is approximated to a certain value with the action of the torsional spring. However, the action of the torsional spring is less considered in the dynamic analysis of the deployable truss antenna using the ANCF description of flexible components, which leads to deviation from practical application in engineering. Therefore, in this work, a mechanics model of the torsional spring is incorporated into the dynamic governing equations derived by the ANCF method to investigate the deployment dynamics of the hoop truss antenna. 
This paper is organized as follows. In Section 2, the dynamic equations of the truss antenna are developed based on the ANCF element. Section 3 establishes an equivalent mechanic model of the torsional spring considering the effect of the torsional spring. In Section 4, the dynamic simulation of the deployment of the truss antenna is presented and we conclude the presented work in Section 5.

\section{Dynamic Modelling of the Truss Antenna}

\subsection{Dynamic Equations of Truss}

The deployable truss consists of a certain amount of parallelogram mechanisms named truss cells, which provide a smooth deployment for the antenna. As illustrated in Figure 2, two adjacent cells comprise of three synchronous joints (point $B_{i}, D_{i}, C_{i+1}$ ), three T-shaped joints (Points $A_{i}, C_{i}, D_{i+1}$ ), horizontal rods, vertical rods, and diagonal sleeves. The truss can be deployed by pulling the cables through the diagonal sleeve after a pre-deployed phase provided by the torsional spring.

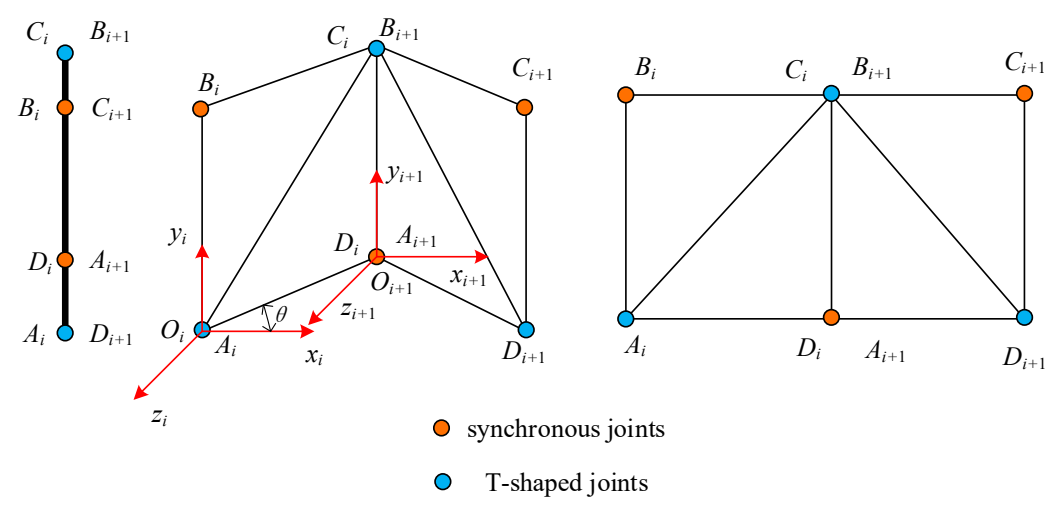

Figure 2. Deployment of the deployable truss.

To describe the motion of the truss, as illustrated in Figure 2, a body coordinate frame of reference $O_{i} x_{i} y_{i} z_{i}$ is attached on the truss cell $i(i=1 \sim n)$, in which axis $O_{i} y_{i}$ coincides with the rod $A_{i} B_{i}$. For a deployable truss consisting of $n$ cells, define $\varphi=2 \pi / n$ as the angle between the coordinate systems $O_{i} x_{i} y_{i} z_{i}$ and $O_{i+1} x_{i+1} y_{i+1} z_{i+1}$. The configuration of truss cell is determined by the deployment angle $\theta$, which is the angle between $A_{i} D_{i}$ and the axis $x_{i}$. Assuming the global coordinate reference is attached to the body frame $O_{1} x_{1} y_{1} z_{1}$, the transformation matrix from the frame $O_{i} x_{i} y_{i} z_{i}$ to $O_{i+1} x_{i+1} y_{i+1} z_{i+1}$ can be formulated as

$$
\mathbf{T}_{i+1}=\left[\begin{array}{cccc}
\cos \varphi & 0 & \sin \varphi & X_{D i} \\
0 & 1 & 0 & Y_{D i} \\
-\sin \varphi & 0 & \cos \varphi & 0 \\
0 & 0 & 0 & 1
\end{array}\right]
$$

where $L_{2}$ is the length of the horizontal rod. Note that the deployment angle $\theta$ is positive when the cell $i$ is odd, while $\theta$ is negative when $i$ is even. $X_{D i}$ and $Y_{D i}$, the translational displacement from $O_{i}$ to $\mathrm{O}_{i+1}$, can be obtained by

$$
\left\{\begin{array}{l}
X_{D i}=L_{2} \cos \theta, Y_{D i}=L_{2} \sin \theta, \quad \text { when } i \text { is odd number } \\
X_{D i}=L_{2} \cos \theta, Y_{D i}=-L_{2} \sin \theta, \quad \text { when } i \text { is even number }
\end{array}\right.
$$

Then, the global position vector of an arbitrary point on the truss cell $i$ can be obtained by transforming the body frame into the global frame and it can be expressed as 


$$
\mathbf{s}=\left[\begin{array}{l}
x \\
y \\
z \\
1
\end{array}\right]=\left[\begin{array}{cccc}
\cos [(i-1) \varphi] & 0 & \sin [(i-1) \varphi] & \left\{\sum_{k=2}^{i} \cos [(k-2) \varphi]\right\} L_{2} \cos \theta \\
0 & 1 & 0 & 0.5\left[1-(-1)^{i-1}\right] L_{2} \sin \theta \\
-\sin [(i-1) \varphi] & 0 & \cos [(i-1) \varphi] & -\left\{\sum_{k=2}^{i} \sin [(k-2) \varphi]\right\} L_{2} \cos \theta \\
0 & 0 & 0 & 1
\end{array}\right] \mathbf{P}_{i}
$$

where $\mathbf{s}$ is the global position vector of the truss and $\mathbf{P}_{i}=\left[P_{i x}, P_{i y}, P_{i z}, 1\right]^{\mathrm{T}}$ is the position vector of the point defined in the body coordinate system.

Velocity vector of the point on the truss can be derived as

$$
\mathbf{v}=\left[\begin{array}{c}
v_{x} \\
v_{y} \\
v_{z}
\end{array}\right]=\left[\begin{array}{c}
-\left\{\sum_{k=2}^{i} \cos [(k-2) \varphi]\right\} L_{2} \dot{\theta} \sin \theta \\
0.5\left[1-(-1)^{i-1}\right] L_{2} \dot{\theta} \cos \theta \\
\left\{\sum_{k=2}^{i} \sin [(k-2) \varphi]\right\} L_{2} \dot{\theta} \sin \theta
\end{array}\right]
$$

Mass of the rod can be equivalent to the nodes of the rod, and ignoring the geopotential energy of the deployable truss considering the working condition, the kinetic energy can be expressed as

$$
T_{t}=m_{t} \sum_{j=2}^{n}\left(v_{x}^{2}+v_{y}^{2}+v_{z}^{2}\right)
$$

where $m_{t}$ is the equivalent mass of the rod.

\subsection{Dynamic Equations of Cable Net}

In this section, the ANCF description of a flexible body is employed to predict the large deformation of cable net during deployment of the deployable antenna. With the metric that the gradient of the node is adopted as node coordinates, the ANCF mesh can precisely capture the flexible deformation as well as rigid motion [16]. Herein, a gradient deficient beam element, as shown in Figure 3, widely used in the application of the cable problem, is selected to discrete the cable net. Each element consists of two nodes with generalized coordinates expressed as

$$
\mathbf{e}=\left[\begin{array}{llll}
\mathbf{r}_{1}^{\mathrm{T}}(0) & \mathbf{r}_{1, x}^{\mathrm{T}}(0) & \mathbf{r}_{2}^{\mathrm{T}}(L) & \mathbf{r}_{2, x}^{\mathrm{T}}(L)
\end{array}\right]^{\mathrm{T}}
$$

where $\mathbf{r}_{i}(i=1,2)$ is the vector of the global position coordinates and $\mathbf{r}_{i, x}$ is the vector of the gradient coordinates defined as $\mathbf{r}_{i, x}=\partial \mathbf{r} / \partial x$. L is the length of element.

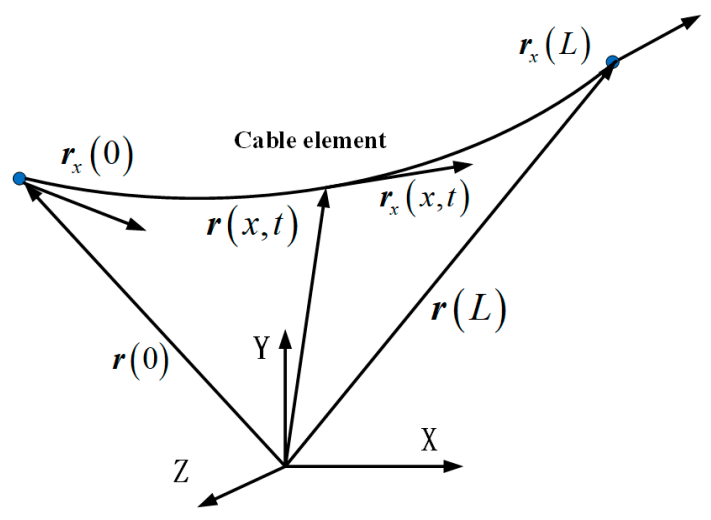

Figure 3. Deployment of the deployable truss. 
Thus, the global position vector of an arbitrary particle on the point is defined as

$$
\mathbf{r}(x, t)=\mathbf{S}(x) \mathbf{e}(t)
$$

where $\mathbf{S}$ is the shape function matrix expressed as

$$
\mathbf{S}(x)=\left[\begin{array}{llll}
S_{1}(x) \mathbf{I}_{3 \times 3} & S_{2}(x) \mathbf{I}_{3 \times 3} & S_{3}(x) \mathbf{I}_{3 \times 3} & S_{4}(x) \mathbf{I}_{3 \times 3}
\end{array}\right]
$$

in which $\mathbf{I}$ is the identity matrix and the items in Equation (8) are as follows

$$
\left\{\begin{array}{l}
S_{1}=1-3 \xi^{2}+2 \xi^{3} \\
S_{2}=L\left(\xi-2 \xi^{2}+\xi^{3}\right) \\
S_{3}=3 \xi^{2}-2 \xi^{3} \\
S_{4}=L\left(-\xi^{2}+\xi^{3}\right)
\end{array}\right.
$$

where $\xi=x / L, L$ is the length of the cable element.

Differentiating of Equation (7) with respect to time, the global velocity vector is obtained as

$$
\dot{\mathbf{r}}(x, t)=\mathbf{S}(x) \dot{\mathbf{e}}(t)
$$

The kinetic energy of the element is formulated as

$$
T_{e}=\frac{1}{2} \int_{0}^{L} \int_{A} \rho \dot{\mathbf{r}} \dot{\mathbf{r}} d A d x=\frac{1}{2} \dot{\mathbf{e}}^{\mathrm{T}} \mathbf{M}_{e} \dot{\mathbf{e}}
$$

where $\rho$ and $A$ denote the density and area of the cross-section. $\mathbf{M}_{e}$ denotes the consistent mass matrix of the cable element that can be clarified as

$$
\mathbf{M}_{e}=m_{e}\left[\begin{array}{cccc}
\frac{13}{35} \mathbf{I} & \frac{11}{210} L \mathbf{I} & \frac{9}{77} \mathbf{I} & -\frac{13}{420} \mathbf{I} \\
& \frac{1}{105} L^{2} \mathbf{I} & \frac{13}{420} L \mathbf{I} & -\frac{1}{140} L^{2} \mathbf{I} \\
& & \frac{13}{35} \mathbf{I} & -\frac{11}{210} L \mathbf{I} \\
s y m & & & \frac{1}{105} L^{2} \mathbf{I}
\end{array}\right]
$$

where $m_{e}$ is the mass of the cable element and $\mathbf{I}$ is the identity matrix.

The strain energy of the ANCF cable element can be obtained with the following formulation

$$
U_{e}=\frac{1}{2} \int_{0}^{L}\left(E A \varepsilon^{2}+E J \kappa^{2}\right) d x
$$

where $E$ is the elastic modulus and $J$ is the second moment of the cross-sectional area. The axial strain $\varepsilon$ and curvature $\mathcal{K}$ are defined by the following expression, respectively.

$$
\begin{gathered}
\varepsilon=\sqrt{\mathbf{r}_{x}^{\mathrm{T}} \mathbf{r}_{x}}-1 \\
\kappa=\frac{\left|\mathbf{r}_{x} \times \mathbf{r}_{x x}\right|}{\left|\mathbf{r}_{x}\right|^{3}}
\end{gathered}
$$

Due to the high nonlinearity of the formula of the strain energy, numerical integration of Gaussian quadrature is adopted in this work. Note that 5 integration points are adopted to evaluate the elastic force considering a good trade-off between computational cost and numerical accuracy.

The total kinetic energy and total strain energy of the cable net can be formulated as

$$
T_{\mathcal{c}}=\sum_{i=1}^{n_{e}} T_{e}, \quad U_{c}=\sum_{i=1}^{n_{e}} U_{e}
$$




\subsection{Dynamic Equations of System}

The total kinetic energy and strain energy of the deployable antenna could be obtained as:

$$
T=T_{t}+T_{c}, \quad U=U_{c}
$$

Thus, dynamic equations of the deployable antenna can be obtained in a compact form using the Lagrangian multiplier method

$$
\left\{\begin{array}{l}
\mathbf{M} \ddot{\mathbf{q}}+\mathbf{C}_{\mathbf{q}}^{T} \boldsymbol{\lambda}+\mathbf{Q}_{k}=\mathbf{Q}_{e} \\
\mathbf{C}(\mathbf{q})=0
\end{array}\right.
$$

with $\mathbf{q}=\left[\begin{array}{ll}\boldsymbol{\theta} & \mathbf{e}\end{array}\right]^{\mathrm{T}}$ being the vector of generalized coordinates, in which $\theta$ is the vector of the deploy angle of each cell. $\mathbf{M}$ is the mass matrix of the antenna system. $\mathbf{Q}_{k}$ and $\mathbf{Q}_{e}$ are the vector of elastic forces and vector of external forces, respectively. $\mathbf{C}$ is the vector of the constraint equations. $\mathbf{C}_{\mathbf{q}}$ is the Jacobian matrix obtained by differentiating the constraint equations with respect to the generalized coordinates. $\lambda$ is the vector of Lagrange multiplier.

\section{Deployment Modelling of Truss Antenna Using Torsional Spring}

To improve the efficiency of deployment, a torsional spring is usually assembled in the synchronous joint to deploy the hoop truss to a certain value of deploy angle. A simplified mechanics model of torsion spring was given in the literature [24], in which the torque varies linearly from 4 to $2 \mathrm{~N} \cdot \mathrm{m}$ during the whole deployment. Assuming that the torsional angle acted by the torsional spring ranges with $\left[0, \alpha_{0}\right]$, then the torque produced by the torsional spring can be expressed as

$$
\tau=K\left(\alpha_{0}-\alpha\right)+c \dot{\alpha}
$$

where $K=128 / 31 \pi \mathrm{Nm} / \mathrm{rad}$ and $c=270 \mathrm{Nms} / \mathrm{rad}$ are the stiffness coefficient and damping coefficient of the torsional spring, respectively. As shown in Figure 4, $\alpha$ is the angle between the horizontal rods and perpendicular rods.

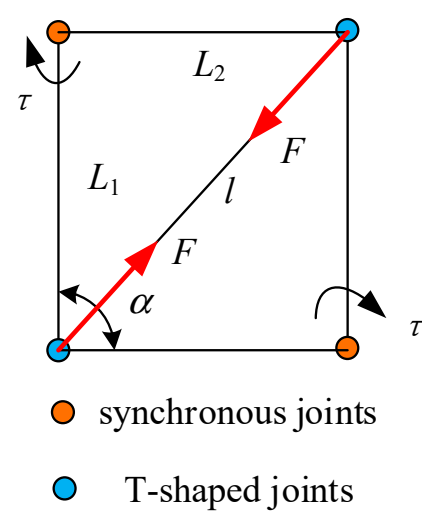

Figure 4. Equivalence of torsional spring.

Defining $F$ as the driving force equivalent to the torque of the torsional spring, as shown in Figure 4, using the principle of virtual work, we have

$$
F \delta l+2 \tau \delta \alpha=0
$$

The virtual change in $l$ can be obtained by variation of $l$ with respect to $\alpha$

$$
\delta l=\frac{-L_{1} L_{2} \sin \alpha}{\sqrt{L_{1}^{2}+L_{2}^{2}+2 L_{1} L_{2} \cos \alpha}} \delta \alpha
$$


Substituting Equation (20) into Equation (19), the torque in Equation (19) is transformed into an equivalent driving force applied on the diagonal rod, expressed as

$$
F=\frac{2 \tau \sqrt{L_{1}^{2}+L_{2}^{2}+2 L_{1} L_{2} \cos \alpha}}{L_{1} L_{2} \sin \alpha}
$$

In order to consider the attenuation of pulling force caused by friction of bearing, Figure 5 schematically depicts a diagram of the pulley. As illustrated in Figure 5, the frictional moment generated by the bearing can be obtained by the formula of Palmgren [24].

$$
M=f F_{\lambda}
$$

where $f=u d$ denotes the frictional coefficient, in which $u$ is the frictional factor and $d$ is the pitch diameter of the bearing. $F_{\lambda}$ is the synthetic moment load acted on the bearing. As for the ball bearing, in this case, $F_{\lambda}$ equals to the radial load $F_{r}[6]$, thus Equation (22) can be written as

$$
M=f F_{r}
$$

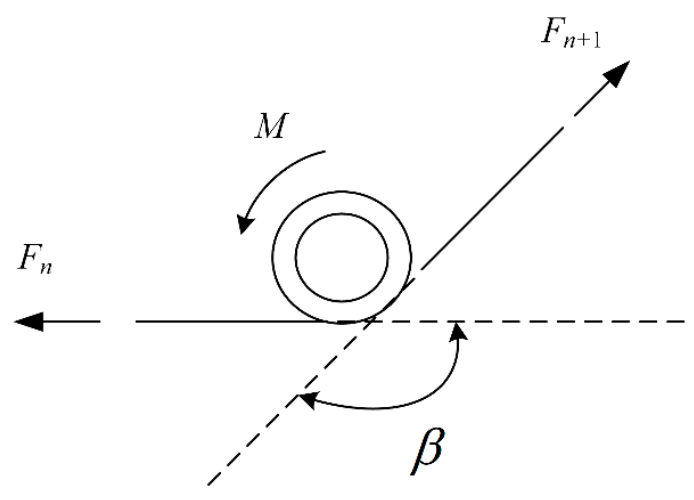

Figure 5. Schematic diagram of the pulley.

Dynamic equations of the pulley can be obtained using the law of motion of the center of mass and theorem of moment of momentum, respectively.

$$
\left\{\begin{array}{l}
F_{n}+F_{n+1}+F_{r}-m_{p} a_{p}=0 \\
R_{n} \times F_{n}+R_{n+1} \times F_{n+1}+M-\frac{1}{2} m_{p}\left|R_{i}\right| 2 \frac{d \omega}{d t}=0
\end{array}\right.
$$

where $m_{p}, a_{p}$, and $\omega$ are the mass, acceleration, and angular velocity of the pulley. $R_{n}$ denotes the vector from the center of the pulley towards $F_{n}$. Considering $a_{p},\left|R_{i}\right|$ and $d \omega / d t$ are very small in Equation (24), which can be transformed as

$$
\left\{\begin{array}{l}
F_{n}+F_{n+1}+F_{r}=0 \\
R_{n} \times F_{n}+R_{n+1} \times F_{n+1}+M=0
\end{array}\right.
$$

According to Equations (24) and (25), one can obtain the following expressions

$$
\left\{\begin{array}{l}
F_{r}^{2}=F_{n}^{2}+F_{n+1}^{2}+2 F_{n} F_{n+1} \cos \beta \\
\left(F_{n}-F_{n+1}\right) R=f F_{r}
\end{array}\right.
$$

Further, Equation (26) can be transformed as

$$
\left(F_{n}-F_{n+1}\right) R-f \sqrt{F_{n}^{2}+F_{n+1}^{2}+2 F_{n} F_{n+1} \cos \beta}=0
$$


The open square item in Equation (27) can be transformed as

$$
\begin{aligned}
F_{n}^{2}+F_{n+1}^{2}+2 F_{n} F_{n+1} \cos \beta= & F_{n}^{2} / 2+F_{n}^{2} / 2+F_{n+1}^{2} / 2+F_{n+1}^{2} / 2+ \\
& F_{n} F_{n+1} \cos \beta / 2+F_{n} F_{n+1} \cos \beta / 2+F_{n} F_{n+1} \cos \beta
\end{aligned}
$$

Considering the fact that the driving force on each side of a pulley is nearly the same, that is $F_{n} \approx F_{n+1}$, one can obtain

$$
\begin{aligned}
F_{n}^{2}+F_{n+1}^{2}+2 F_{n} F_{n+1} \cos \beta \approx & F_{n}^{2} / 2+F_{n} F_{n+1} / 2+F_{n+1}^{2} / 2+F_{n} F_{n+1} / 2+ \\
& F_{n}^{2} \cos \beta / 2+F_{n+1}^{2} \cos \beta / 2+F_{n} F_{n+1} \cos \beta
\end{aligned}
$$

Simplifying Equation (29), it can be expressed as

$$
\begin{aligned}
F_{n}^{2}+F_{n+1}^{2}+2 F_{n} F_{n+1} \cos \beta & \approx F_{n}^{2} \frac{1+\cos \beta}{2}+F_{n+1}^{2} \frac{1+\cos \beta}{2}+2 F_{n} F_{n+1} \frac{1+\cos \beta}{2} \\
& =\left(F_{n} \cos \frac{\beta}{2}+F_{n+1} \cos \frac{\beta}{2}\right)^{2}
\end{aligned}
$$

Thus, substituting Equation (30) into Equation (27), one can obtain the attenuation formulation of driving force

$$
F_{n+1}=\frac{R-f \cos (\beta / 2)}{R+f \cos (\beta / 2)} F_{n}
$$

where $R$ is the radius of pulley and $\beta$ is the angle between the driving cables. From Equation (31), it can be found that the attenuation coefficient is related with the $\beta$ and radius of pulley $R$, which implies that with the augment in $R$, the attenuation coefficient increases as well.

Substituting the equivalent driving force of the torsional spring into the dynamic Equation (17) in the generalized form, dynamic simulation of the mesh reflector antennas' deployment can be performed.

\section{Dynamics Simulation of Truss Antenna}

In this section, dynamic simulation of the deployable truss antenna is performed numerically. It is noted that the bending curvatures in Equation (13) are less dominant than the axial strain in cable dynamics. Therefore, the bending deformation of the cable element is neglected in the dynamic simulation. Additionally, in order to obtain the initial configuration with a shrinking process of the truss, a small elastic modulus for the cable is adopted to enhance the simulation efficiency with an integration step as large as possible. The cable net is discretized with 1650 cable elements and Table 1 lists the parameters used in the numerical simulation.

Table 1. Parameters used in simulation.

\begin{tabular}{cccc}
\hline Parameters & Value & Parameters & Value \\
\hline Length of horizontal rods & $1 \mathrm{~m}$ & Truss cell number & 12 \\
Length of vertical rods & $1.2 \mathrm{~m}$ & Density of rod & $2700 \mathrm{Kg} / \mathrm{m}^{3}$ \\
Diameter of rod & $0.025 \mathrm{~m}$ & Elastic modulus of rod & $75 \mathrm{Gpa}$ \\
Diameter of cable net & $0.005 \mathrm{~m}$ & Cable density & $1430 \mathrm{Kg} / \mathrm{m}^{3}$ \\
Number of cable elements & 1650 & Cable elastic modulus & $12 \mathrm{Mpa}$ \\
\hline
\end{tabular}

Figure 6 presents the deployment configuration associated with different deployed moment, in which an asynchronous deployment along the anticlockwise direction is clearly observed. The diameter of the truss is deployed from an initial 8 to $30 \mathrm{~m}$ for the final configuration. It should be noted that due to the complicated property of the initial configuration, the fully deployed configuration of the truss antenna using the ANCF cable element is established firstly. Then, under the action of identical driving velocity of the parallelogram mechanisms, the initial configuration of the antenna is obtained by shrinking the fully deployed configuration to a shrinkable quasi-static configuration [27]. 


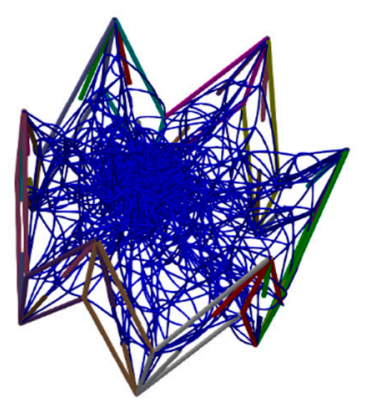

(a)

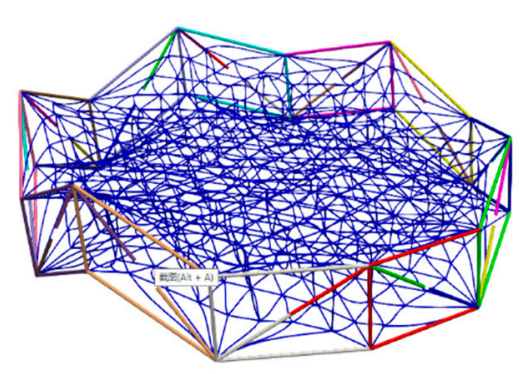

(c)

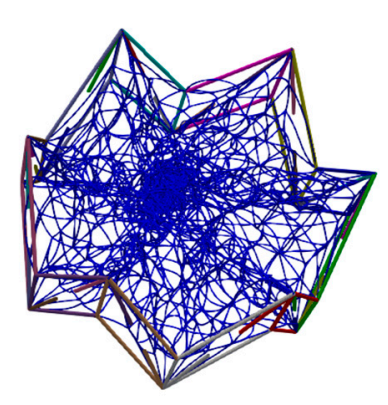

(b)

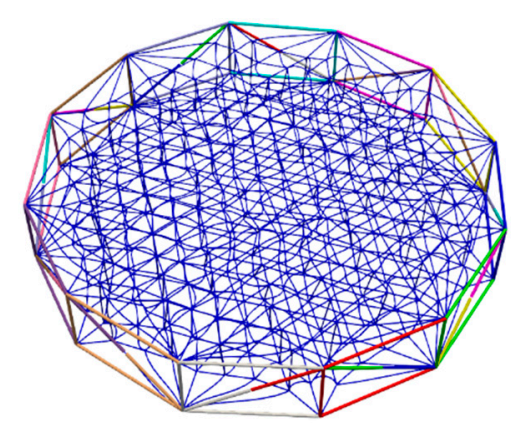

(d)

Figure 6. Deployment configuration. (a) $t=0.2 \mathrm{~s}$, (b) $t=1.2 \mathrm{~s}$, (c) $t=3.1 \mathrm{~s}$ (d) $t=4.1 \mathrm{~s}$.

The variation of the relative distance of the diagonal rods is illustrated in Figure 7. It can be seen that the deviation between different cells increases with the deployment of truss. In the initial deployed phase, each cell deploys synchronously with the action of the torque of the torsional spring. The driving force from a motor acts when the torque of the torsional spring is worked out, which leads to an asynchronous deployment caused by the attenuation of driving force.

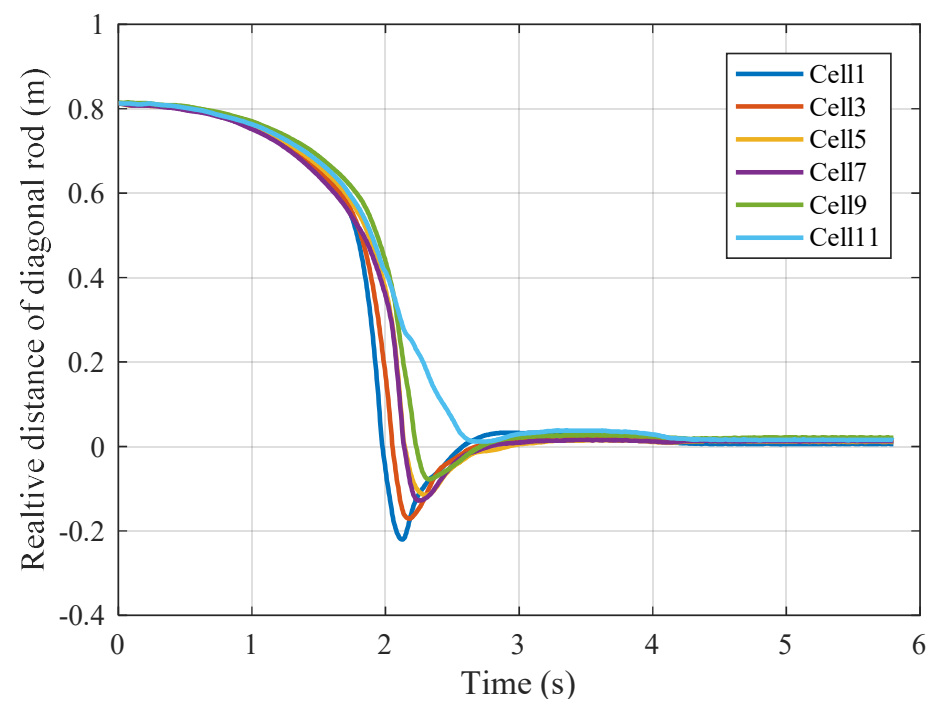

Figure 7. Relative distance of the diagonal rod.

Figure 8 presents the variation of the deployment angle, which implies the phenomenon of asynchronous deployment as well. As a point of attention, the truss cell 12 reaches the final lock place more slowly than other truss cells, which indicates that the driving force acting on the diagonal 
rod of cell 12 is affected by attenuation intensely. The fact that asynchronous deployment is caused by the attenuation of the driving forces can also be found in the literature [27], which indicates the effectiveness of the established dynamic model of the deployable antenna system.

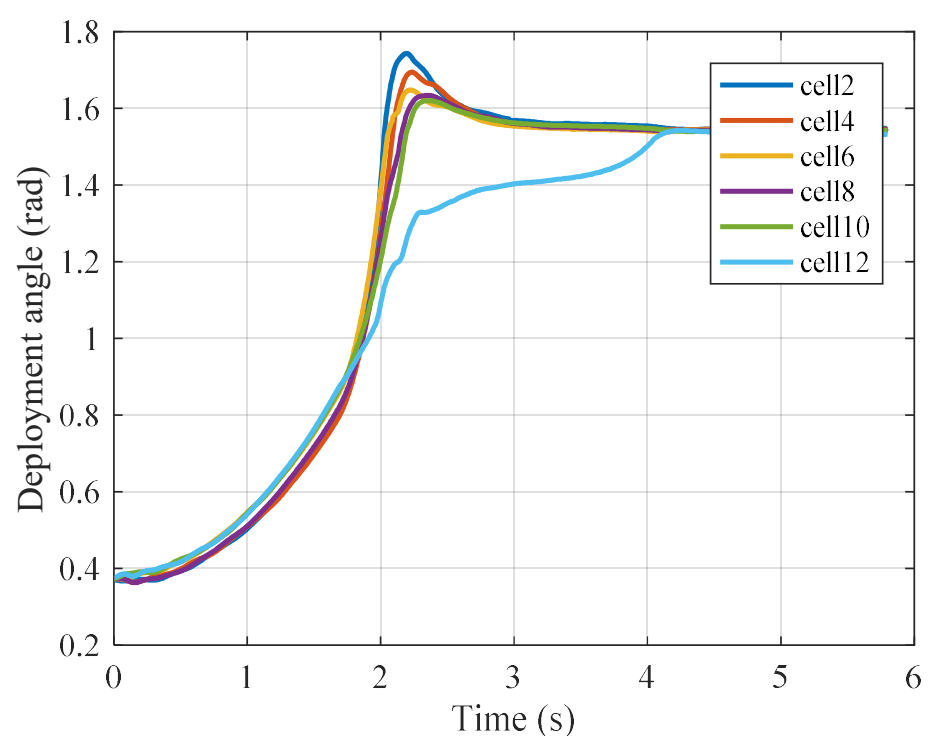

Figure 8. Deployment angle.

Furthermore, for a presentative point, the tensions of both the cable net and the tensile cable are obtained by the average value of element tensions, respectively. Figure 9 exhibits the time history response of the mean tension of the tensile cable; a rapid augment in tension from 1 to $6 \mathrm{~N}$ appears around the moment $t=2 \mathrm{~s}$, when the antenna is deployed to the final configuration. Additionally, clear oscillation can be observed during the whole deployment. Variation of the mean tension of the cable net is displayed in Figure 10. Different from the amplitude of tension of the tensile cable in Figure 10, the tension of the cable net increases to $25 \mathrm{~N}$ and with a more intense oscillation. It is noted that the behavior of the rapid increase in amplitude of the cable tension in the phase of post-deployment is in line with the conclusions in the literature [29].

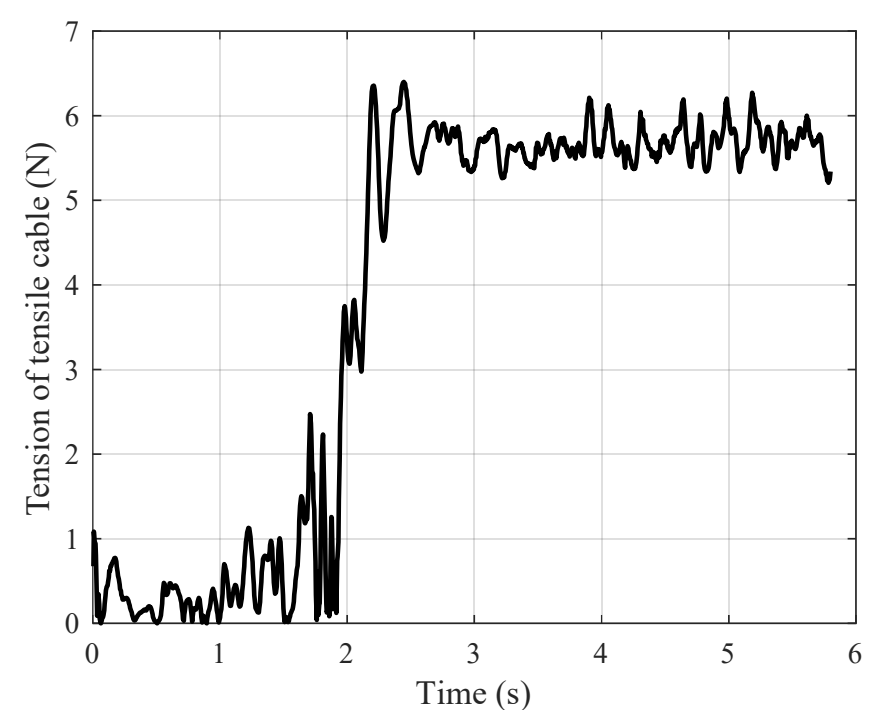

Figure 9. Tension of tensile cable. 


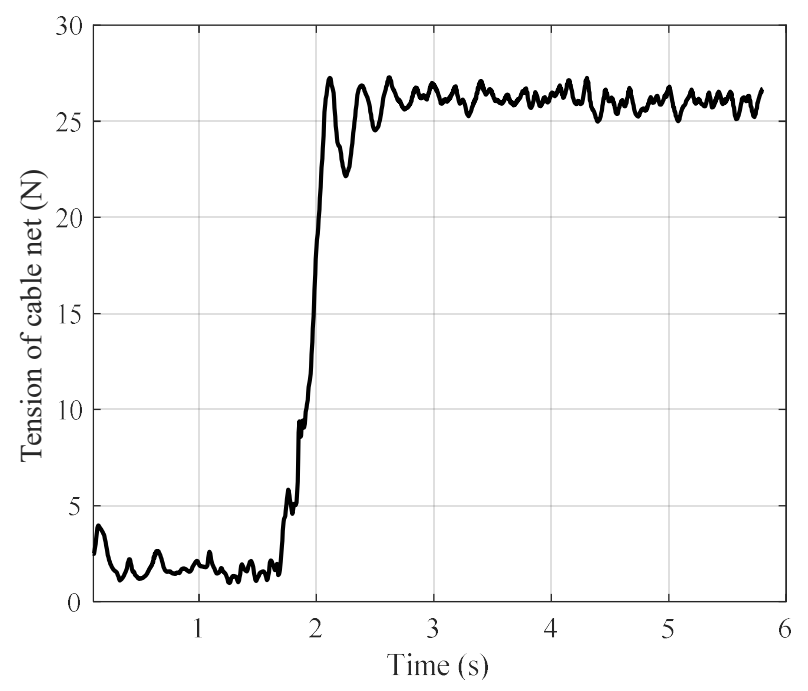

Figure 10. Tension of cable net.

The velocity responses of the cable nodes that are located in Figure 11 are presented in Figures 12 and 13, respectively. A phenomenon of significant shaking is observed during the deployment. Considering that node 1 is farther from the center of the net than node 2, it can be concluded that with the distance of the nodes to the center of the net increasing, the shaking of velocity responses become more intense.

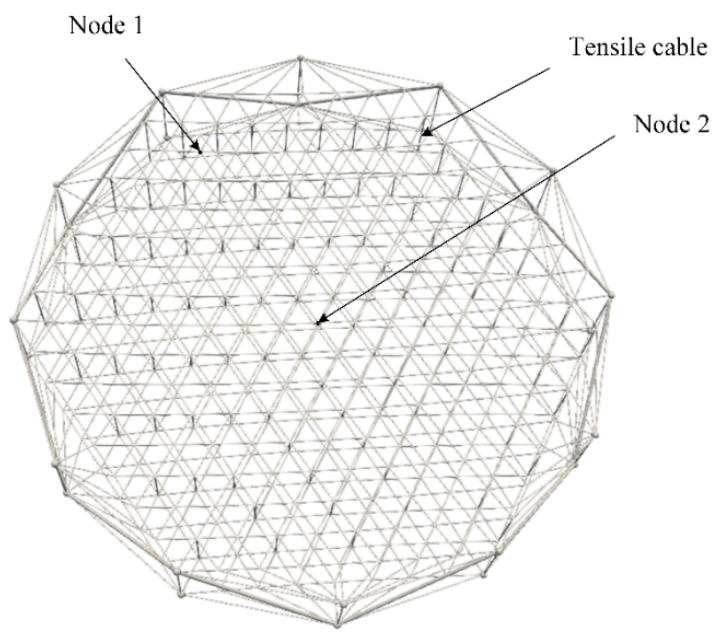

Figure 11. Location of Node 1 and Node 2. 


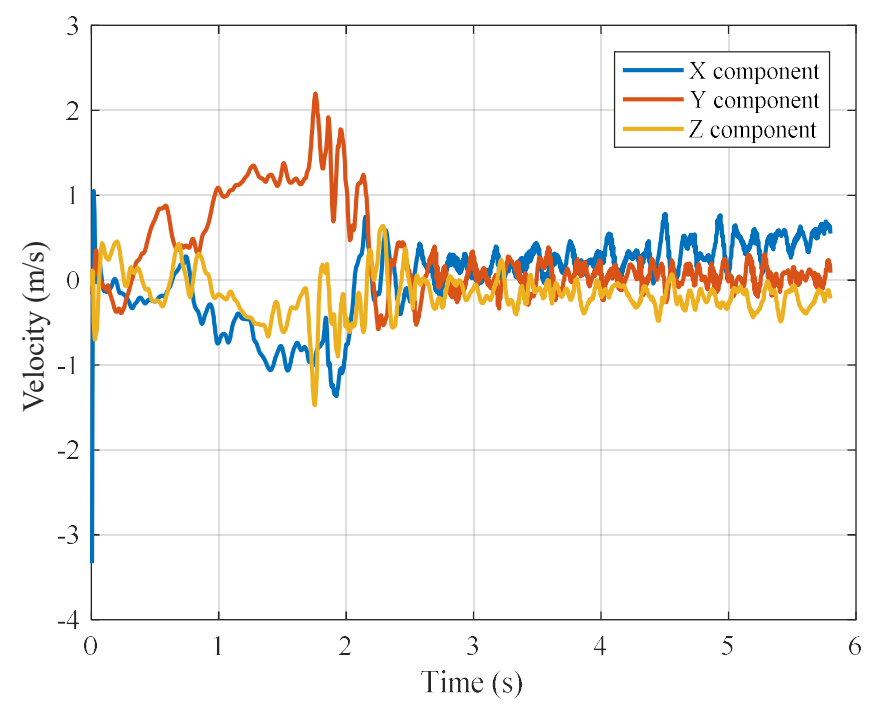

Figure 12. Velocity response of Node 1.

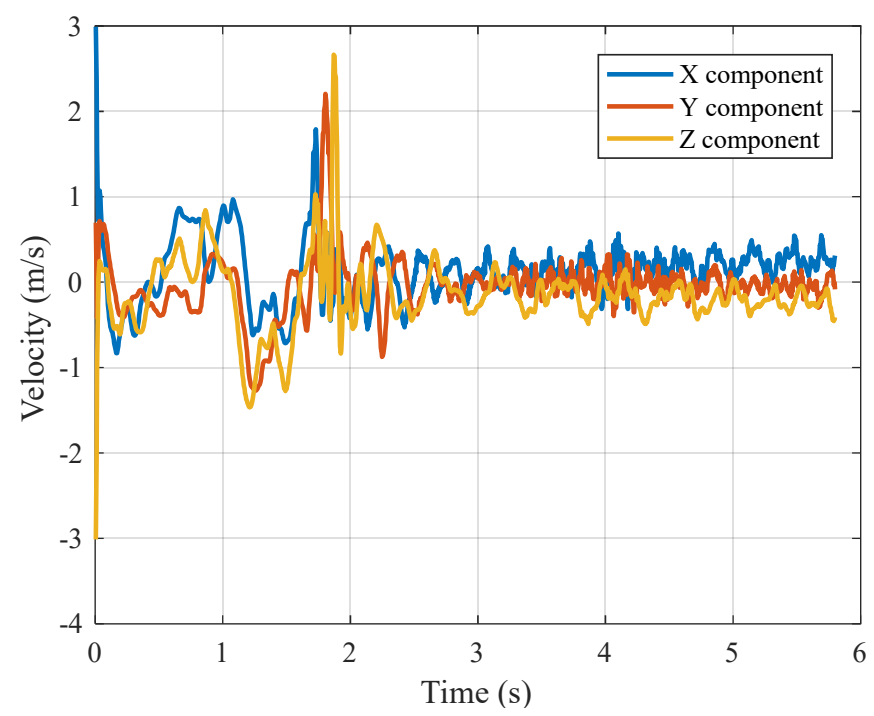

Figure 13. Velocity response of Node 2.

\section{Conclusions}

The presented work aims at the analysis of the deployment dynamics of a hoop truss considering the effect of the torsional spring and a time-varying attenuation coefficient of driving force. With the employment of the ANCF method, large deformation of the cable net during deployment of the deployable antenna can be predicted accurately. Based on the derived dynamic governing equations, dynamic simulation can be performed numerically. From the results obtained, the phenomenon of unsynchronized deployment during the latter phase can be observed, which results from the attenuation of driving force caused by the friction effect of the pulley. Furthermore, with the increase in the deployable angle, the tension of the cable net increases with oscillation. Particularly, an intense augment in cable tension arises in the final phase of deployment, which implies that the driving force affects the tension significantly. Additionally, the impact force induced by the lock mechanism will exert an influence on the tension of the cable net. The flexibility of the truss is neglected in this study; further investigation considering the effect of the flexible rod should be conducted in the future.

Author Contributions: X.J. conceived the proposal of the paper, performed the simulation, and analyzed the data. Z.B. provided guidance, key suggestions, and finalized the paper. All authors have read and agreed to the published version of the manuscript. 
Funding: This research received no external funding.

Conflicts of Interest: The authors declare no conflict of interest.

\section{Nomenclature}

\begin{tabular}{|c|c|}
\hline$A$ & cross-sectional area of cable \\
\hline$c$ & damping coefficient of the torsional spring \\
\hline C & vector of constraint equations \\
\hline $\mathrm{C}_{\mathrm{q}}$ & Jacobian matrix of the constraint equations \\
\hline e & generalized coordinates of cable element \\
\hline E & elastic modulus of cable \\
\hline$f$ & frictional coefficient \\
\hline$F$ & equivalent driving force \\
\hline$F_{n}, F_{n+1}$ & driving forces on the pulley \\
\hline$F_{\lambda}$ & synthetic moment load \\
\hline$F_{r}$ & radial force of the bearing \\
\hline$i$ & clockwise number of the cell \\
\hline I & identity matrix \\
\hline K & stiffness coefficient of the torsional spring \\
\hline$L_{1}$ & length of the vertical rod \\
\hline$L_{2}$ & length of the horizontal rod \\
\hline$L$ & length of the cable element \\
\hline$m_{t}$ & equivalent mass of the truss cell \\
\hline$m_{e}$ & mass of the cable element \\
\hline$m_{p}$ & mass of the pulley \\
\hline $\mathbf{M}$ & mass matrix of the antenna system \\
\hline $\mathbf{M}_{e}$ & mass matrix of the cable element \\
\hline M & frictional moment \\
\hline$n$ & number of the truss cells \\
\hline q & vector of generalized coordinates of antenna \\
\hline$U_{e}$ & strain energy of the cable element \\
\hline$U_{c}$ & strain energy of the cable \\
\hline $\mathbf{v}$ & global velocity vector of the truss \\
\hline $\mathbf{P}_{i}$ & local position vector of the truss \\
\hline $\mathbf{Q}_{e}$ & vector of external forces \\
\hline $\mathbf{r}$ & global position vector of cable element \\
\hline$R_{n}, R_{n+1}$ & driving force from the center of pulley to the action point \\
\hline $\mathbf{s}$ & global position vector of the truss \\
\hline $\mathbf{S}$ & shape function matrix of cable element \\
\hline$T_{t}$ & kinetic energy of the truss \\
\hline$T_{e}$ & kinetic energy of the cable element \\
\hline$\alpha$ & angle between horizontal rod and perpendicular rod \\
\hline$\varphi$ & angle between two body coordinate frames \\
\hline$\theta$ & deployment angle \\
\hline$\theta$ & Vector of the generalized coordinates \\
\hline$\lambda$ & Lagrange multiplier \\
\hline$\tau$ & torque of the torsional spring \\
\hline
\end{tabular}

\section{References}

1. Shi, C.; Guo, H.; Zheng, Z.; Li, M.; Liu, R.; Deng, Z. Conceptual configuration synthesis and topology structure analysis of double-layer hoop deployable antenna unit. Mech. Mach. Theory 2018, 129, 232-260.

2. Puig, L.; Barton, A.; Rando, N. A review on large deployable structures for astrophysics missions. Acta Astronaut. 2010, 67, 12-26. [CrossRef] 
3. Thomson, M.W. The astromesh deployable reflector. In Proceedings of the IEEE Antennas \& Propagation Society International Symposium, Orlando, FL, USA, 11-16 July 1999; pp. 1516-1519.

4. Meguro, A.; Harada, S.; Watanabe, M. Key technologies for high-accuracy large mesh antenna reflectors. Acta Astronaut. 2003, 53, 899-908. [CrossRef]

5. Sun, Y.; Zhang, W.; Wu, R. Analysis on nonlinear dynamics of circular truss antennae in 6D systems. Appl. Math. Mech. 2019, 40, 282-301.

6. Liu, L.; Shan, J.; Zhang, Y. Dynamics modeling and analysis of spacecraft with large deployable hoop-truss antenna. J. Spacecr. Rocket. 2016, 53, 471-479. [CrossRef]

7. Guo, H.W.; Zhang, J.; Liu, R.Q.; Deng, Z.Q.; Cao, D.Q. Nonlinear dynamics analysis of joint dominated space deployable truss structures. J. Harbin Inst. Technol. 2013, 20, 68-74.

8. Jin, S.A.M. Deployment analysis of large space antenna using flexible multibody dynamics simulation. Acta Astronaut. 2000, 47, 19-26.

9. Liang, C.; Guan, F.; Zhang, H. Deployment experiment of deployable truss antenna. Chin. J. Sp. Sci. 2010, 30, 79-84.

10. Li, P.; Ma, Q.; Song, Y.; Liu, C.; Tian, Q.; Ma, S.; Hu, H. Deployment dynamics simulation and ground test of a large space hoop truss antenna reflector. Sci. Sin. Phys. Mech. Astron. 2017, 47, 104602. [CrossRef]

11. Li, T. Deployment analysis and control of deployable space antenna. Aerosp. Sci. Technol. 2012, 18, 42-47. [CrossRef]

12. Lv, Y.; Shi, Q.; Wang, G.; Liu, F.; Xu, Y.; Li, G.; Zou, X.J. Dynamic finite-element simulation on the phased array radar antenna multiplex damage by fragment and shock wave. In Proceedings of the 2012 International Conference on Quality, Reliability, Risk, Maintenance, and Safety Engineering, Chengdu, China, 15-18 June 2012; pp. 929-933.

13. Zhang, Y.; Li, N.; Yang, G.; Ru, W. Dynamic analysis of the deployment for mesh reflector deployable antennas with the cable-net structure. Acta Astronaut. 2017, 131, 182-189. [CrossRef]

14. Nie, R.; He, B.; Zhang, L.; Fang, Y. Deployment analysis for space cable net structures with varying topologies and parameters. Aerosp. Sci. Technol. 2017, 68,1-10. [CrossRef]

15. Cammarata, A.; Sinatra, R.; Rigano, A.; Lombardo, M.; Maddio, P.D. Design of a large deployable reflector opening system. Machines 2020, 8, 7. [CrossRef]

16. Shabana, A.A.; Mikkola, A.M. Use of the finite element absolute nodal coordinate formulation in modeling slope discontinuity. J. Mech. Des. 2003, 125, 357-387. [CrossRef]

17. Li, B.; Qi, X.; Huang, H.; Xu, W. Modeling and analysis of deployment dynamics for a novel ring mechanism. Acta Astronaut. 2016, 120, 59-74. [CrossRef]

18. Ambrósio, J.A.C.; Neto, M.A.; Leal, R.P. Optimization of a complex flexible multibody systems with composite materials. Multibody Syst. Dyn. 2007, 18, 117-144. [CrossRef]

19. Masashi, I. Effects of coordinate system on the accuracy of corotational formulation for Bernoulli-Euler's beam. Int. J. Solids Struct. 1994, 31, 2793-2806. [CrossRef]

20. Simo, J.C.; Fox, D.D.; Rifai, M.S. On a stress resultant geometrically exact shell model. Part I: Formulation and optimal parametrization. Comput. Methods Appl. Mech. Eng. 1989, 73, 53-92. [CrossRef]

21. Hughes, T.J.R.; Cottrell, J.A.; Bazilevs, Y. Isogeometric analysis: CAD, finite elements, NURBS, exact geometry and mesh refinement. Comput. Methods Appl. Mech. Eng. 2005, 194, 4135-4195. [CrossRef]

22. Shabana, A.A.; Christensen, A.P. Three-dimensional absolute nodal co-ordinate formulation: Plate problem. Int. J. Numer. Methods Eng. 1997, 40, 2775-2790. [CrossRef]

23. Li, T.; Wang, Y. Deployment dynamic analysis of deployable antennas considering thermal effect. Aerosp. Sci. Technol. 2009, 13, 210-215. [CrossRef]

24. Tao, C.; Liu, L.; Zhou, Z.; Tian, Q.; Xing, Z. Analysis on non-synchronous deployment of hoop truss antenna (in chinese). Chin. Sp. Sci. Technol. 2015, 35, 1-8.

25. Liu, L.; Shan, J.; Tao, C.; Cao, P.; Zhou, Z. Simulation of non-synchronous deployment of the large deployable hoop truss antenna. In Proceedings of the 2nd AIAA Spacecraft Structures Conference, Kissimmee, FL, USA, 5-9 January 2015.

26. Lu, S.; Qi, X.; Hu, Y.; Li, B.; Zhang, J. Deployment dynamics of large space antenna and supporting arms. IEEE Access 2019, 7, 69922-69935. [CrossRef]

27. Li, P.; Liu, C.; Tian, Q.; Hu, H.; Song, Y. Dynamics of a deployable mesh reflector of satellite antenna: Parallel computation and deployment simulation. J. Comput. Nonlinear Dyn. 2016, 11, 61005. [CrossRef] 
28. Peng, Y.; Zhao, Z.; Zhou, M.; He, J.; Yang, J.; Xiao, Y. Flexible multibody model and the dynamics of the deployment of mesh antennas. J. Guid. Control Dyn. 2017, 40, 1499-1506. [CrossRef]

29. Du, X.; Du, J.; Bao, H.; Sun, G. Deployment analysis of deployable antennas considering cable net and truss flexibility. Aerosp. Sci. Technol. 2018, 82-83, 557-565. [CrossRef]

Publisher's Note: MDPI stays neutral with regard to jurisdictional claims in published maps and institutional affiliations.

(C) 2020 by the authors. Licensee MDPI, Basel, Switzerland. This article is an open access article distributed under the terms and conditions of the Creative Commons Attribution (CC BY) license (http://creativecommons.org/licenses/by/4.0/). 